\title{
ANÁLISIS DEL CRECIMIENTO Y LA REGENERACIÓN EN RESPUESTA A TRATAMIENTOS SILVÍCOLAS EN BOSQUE NATIVO: ALTERNATIVAS PRODUCTIVAS MÁS SUSTENTABLES ASOCIADAS A LINGUE (Persea lingue (R. et P.) Nees ex Kopp
}

Pinilla, Juan Carlos ${ }^{8}$; Luengo, Karina; Navarrete, Mauricio y Larraín, Oscar

\section{RESUMEN}

Se analiza y describe dasométricamente a tres rodales naturales de lingue ubicados en la región del Bio Bio; dos de ellos en la zona de Nacimiento y un tercero en las cercanías de Coihueco. Se presenta una descripción general de los rodales estudiados, para conformar una visión global de estas formaciones vegetales, describiendo su estructura horizontal, vertical, diamétrica y composición de especies.

Se analiza también un ensayo de espaciamiento, tipo de contenedor y protección de lingue, establecido en Cañete, el cual tiene por objetivo dilucidar el efecto del espaciamiento inicial y de protectores de malla sobre la supervivencia y desarrollo de las plantas.

Palabras Clave: Lingue, caracterización de rodales naturales, supervivencia, crecimiento inicial de plantaciones

\section{SUMMARY}

Three natural stands of lingue located in the Bio Bio region are analyzed and dasometrically described; two of them in the area of Nacimiento and a third one near Coihueco. A general description of the stands studied is presented to form a global vision of these plant formations, describing their species composition and their horizontal, vertical and diametrical structure.

A trial established in Cañete, which aims to elucidate the effect of initial spacing and mesh protectors on plant survival and development, is also analyzed.

Keywords: Lingue, characterization of natural stands, plantation's survival and early growth.

\footnotetext{
${ }^{8}$ Investigadores Instituto Forestal sede Bio Bio, Camino a Coronel Km. 7,5, Concepción. jpinilla@infor.cl
} 


\section{INTRODUCCIÓN}

Es reconocido que el bosque nativo en Chile ha sido degradado por distintos factores, de modo que su calidad productiva es normalmente baja en términos de biomasa total/superficie. Sin embargo, bajo condiciones apropiadas de manejo y aplicación de técnicas silviculturales se puede rejuvenecer el bosque y las especies que lo constituyen, escoger los mejores árboles y mejorar de forma notable la calidad y crecimiento de estos. Para tal efecto, las intervenciones que se realicen en el bosque dependerán de su estructura, fase de desarrollo, las especies principales y secundarias que lo componen, y las condiciones en las que se encuentran, entre otras.

Estas intervenciones deben basarse en la ecología de las especies y la dinámica del bosque que privilegian las especies mejor adaptadas al lugar desde un punto de vista económico y biológico, buscando el mejor ajuste entre los propósitos de manejo del propietario y las limitaciones naturales impuestas por el sitio.

Al respecto, el presente artículo se enmarca en un programa de mediano y largo plazo, centrado en la generación y transferencia de nuevos conocimientos y técnicas silvícolas para el manejo forestal sustentable del bosque nativo y las especies que lo componen.

Particularmente, el documento presenta antecedentes de la especie lingue (Persea lingue (R. et P.) Nees ex Kopp) que se desarrolla en los bosques de Nothofagus de la Región del Bio Bio.

El objetivo es fomentar nuevos conocimientos en técnicas silvícolas y de manejo forestal, de modo que propietarios y comunidades que habitan en los ecosistemas donde habita esta especie recuperen e incremente la provisión sustentable de bienes y servicios relacionados con esta, preferentemente los relacionados con su madera de alto valor (Pinilla y Navarrete, 2013; Pinilla et al., 2015).

\section{METODOLOGÍA}

Se analiza y describe dasométricamente a tres rodales naturales de lingue ubicados en la región del Bio Bio; dos de ellos en la zona de Nacimiento y un tercero en las cercanías de Coihueco. Se presenta una descripción general de los rodales estudiados, para conformar una visión global de estas formaciones vegetales, describiendo su estructura horizontal, vertical, diamétricas y composición de especies. Para la descripción se entrega la estructura diamétrica del bosque y la estructura por especie a modo de lograr una mejor descripción e interpretación de lo que está ocurriendo en estos bosques.

En cuanto a la descripción de la estructura cuantitativa, se entregan dos descripciones, por un lado se describe la composición del renoval, tanto en participación de especies como en participación en área basal en porcentaje, y por otro lado se entrega la información dasométrica promedio en una tabla de rodal en donde se describe para cada clase diamétrica existente las características de cada especie que conforma el rodal, esto en términos de número de árboles por hectárea, altura total $(\mathrm{m})$, diámetro medio cuadrático $(\mathrm{cm})$, área basal $\left(\mathrm{m}^{2}\right)$ y volumen $\left(\mathrm{m}^{3} / \mathrm{ha}\right)$.

Se analiza también un ensayo de espaciamiento y protección de lingue, establecido en Cañete, el cual tiene por objetivo dilucidar el efecto del tamaño de contenedor utilizados para la producción de plantas, espaciamiento de plantación y uso de malla protectora sobre la supervivencia y desarrollo de las plantas. Para este efecto se evalúa una medición de altura, diámetro y supervivencia de las plantas que componen el ensayo y se efectúa el análisis estadístico descriptivo y el análisis de varianza del mismo. La medición evaluada corresponde a la efectuada en 2019, a la edad de cinco años. 


\section{RESULTADOS}

\section{Evaluación de Rodales Naturales de Lingue}

Para el estudio del lingue y de sus opciones de manejo es necesario evaluar rodales naturales que representen diferentes composiciones de edad, características y ubicación. En este contexto, en los puntos siguientes se describen y analizan tres de tales rodales.

\section{Rodal 1. Nacimiento}

Corresponde a un renoval puro de lingue, de aproximadamente 30 años, con baja presencia de avellano (Gevuina avellana Mol.), laurel (Laurelia sempervirens (Ruiz \& Pav.) Tul), peumo (Cryptocarya alba (Molina) Looser) y roble (Nothofagus obliqua (Mirb.) Oerst.), que se ubica en el sector Mitrihue-Los aromos, accesible desde el cruce Santa Lucía de la Ruta Santa JuanaNacimiento. Presenta una estructura coetánea, que probablemente corresponda a una antigua tala rasa y posterior quema de un remanente de bosque Roble-Laurel-Lingue, donde la mayor parte de la regeneración fue consumida por el ganado, excepto la de lingue que debido a su menor palatabilidad fue capaz de regenerar en un solo pulso de establecimiento y desarrollarse hasta alcanzar la estructura actual.

En el rodal se aprecia una gran cantidad de vástagos de Lingue por cada cepa $(4-13)$, lo cual fortalece la hipótesis de la tala rasa y posterior quema. Se obtuvo una frecuencia cercana a los 940 tocones por hectárea, los que, considerando la gran cantidad de retoños por tocón, alcanza a los 7.260 fustes por hectárea (Figura $N^{\circ} 1$ ). Las especies presentes en el rodal, el número de fustes y la participación porcentual según especie se presenta en el Cuadro $N^{\circ} 1$, donde se destaca la mayoritaria presencia de lingue.

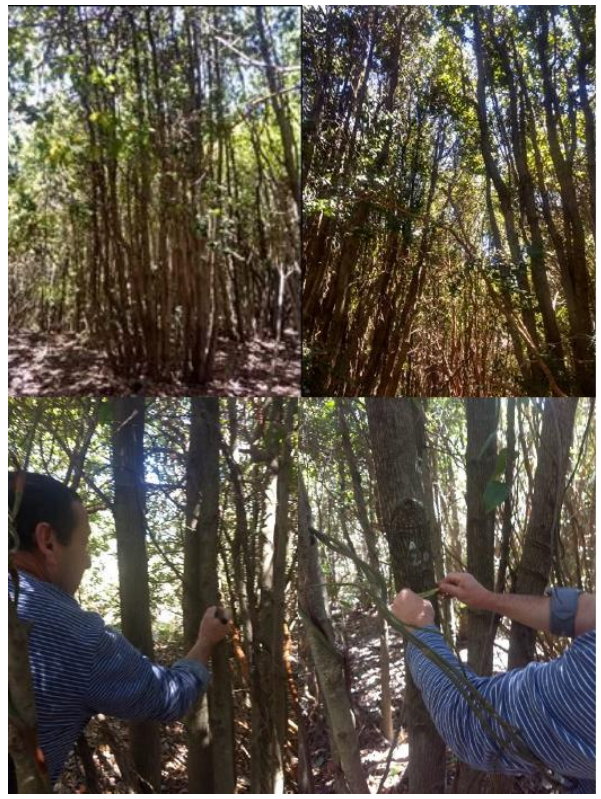

Figura $\mathrm{N}^{\circ} 1$

VISTA DE LA ABUNDANTE RETOÑACION PRESENTE EN RODAL DE NACIMIENTO 1 
Cuadro $\mathrm{N}^{\circ} 1$

ESPECIES PRESENTES EN EL RODAL NACIMIENTO 1 NÚMERO DE FUSTES Y PARTICIPACIÓN SEGÚN ESPECIE

\begin{tabular}{|l|r|r|}
\hline \multirow{2}{*}{ Especie } & \multicolumn{2}{|c|}{ Fustes } \\
\cline { 2 - 3 } & (No/ha) & (\%) \\
\hline Arrayán & 390 & 5 \\
Avellanillo & 100 & 1 \\
Avellano & 50 & 1 \\
Boldo & 30 & 0 \\
Lingue & 6.430 & 89 \\
Maqui & 110 & 2 \\
Roble & 150 & 2 \\
\hline Total & $\mathbf{7 . 2 6 0}$ & $\mathbf{1 0 0}$ \\
\hline
\end{tabular}

El rodal es coetáneo y se encuentra en estado de estancamiento del crecimiento, lo que se aprecia en la mortalidad natural, cruce de copas y su crecimiento.

Los valores medios de DAP, altura y área basal por especie se presentan en el Cuadro $\mathrm{N}^{\circ} 2$, la distribución diamétrica por especie se resume en la Figura $\mathrm{N}^{\circ} 2$ y la relación diámetroaltura en la Figura $\mathrm{N}^{\circ} 3$.

\section{Cuadro $\mathrm{N}^{\circ} 2$}

VALORES DE ALTURA, DAP Y AREA BASAL RODAL NACIMIENTO 1

\begin{tabular}{|l|r|r|r|}
\hline Especie & \multicolumn{1}{|c|}{$\begin{array}{c}\text { DAP } \\
\text { (cm) }\end{array}$} & $\begin{array}{c}\text { Altura } \\
\text { (m) }\end{array}$ & $\begin{array}{c}\text { Área Basal } \\
\left(\mathbf{m}^{\mathbf{2}} \mathbf{/ h a}\right)\end{array}$ \\
\hline Arrayán & 4,4 & 6,4 & 0,58 \\
Avellanillo & 11,6 & 12,7 & 1,06 \\
Avellano & 10,6 & 7,1 & 0,44 \\
Boldo & 7,5 & 9,0 & 0,13 \\
Lingue & 7,0 & 9,3 & 24,74 \\
Maqui & 8,4 & 7,4 & 0,61 \\
Roble & 23,4 & 18,4 & 6,46 \\
\hline Total & \multicolumn{2}{|r|}{} & 34,02 \\
\hline
\end{tabular}

En general el rodal destaca por la predominancia de lingue en términos de número de árboles y área basal, destacándose también la mayor envergadura de los individuos de roble, los cuales corresponderían a remanentes del bosque original. 


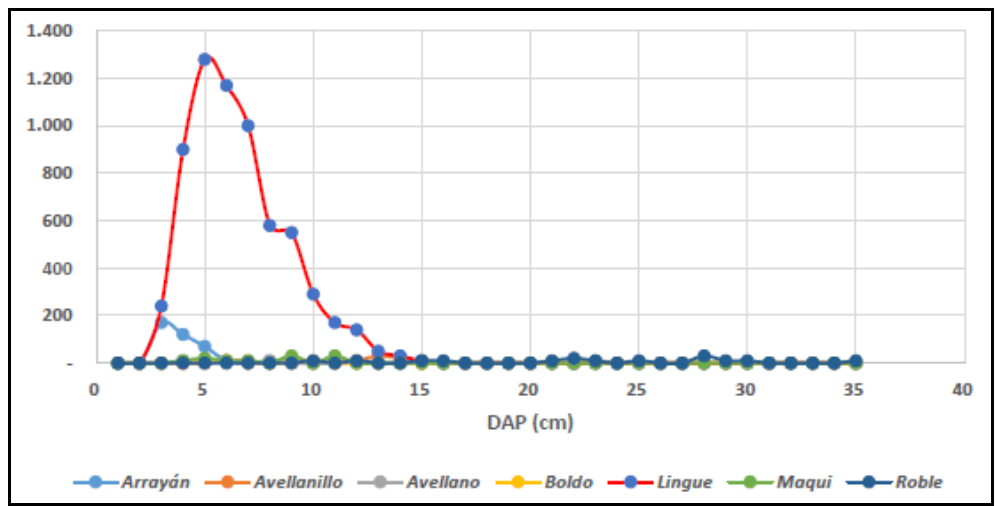

Figura $N^{\circ} 2$

DISTRIBUCIÓN DIAMÉTRICA DE LOS ÁRBOLES RODAL NACIMIENTO 1

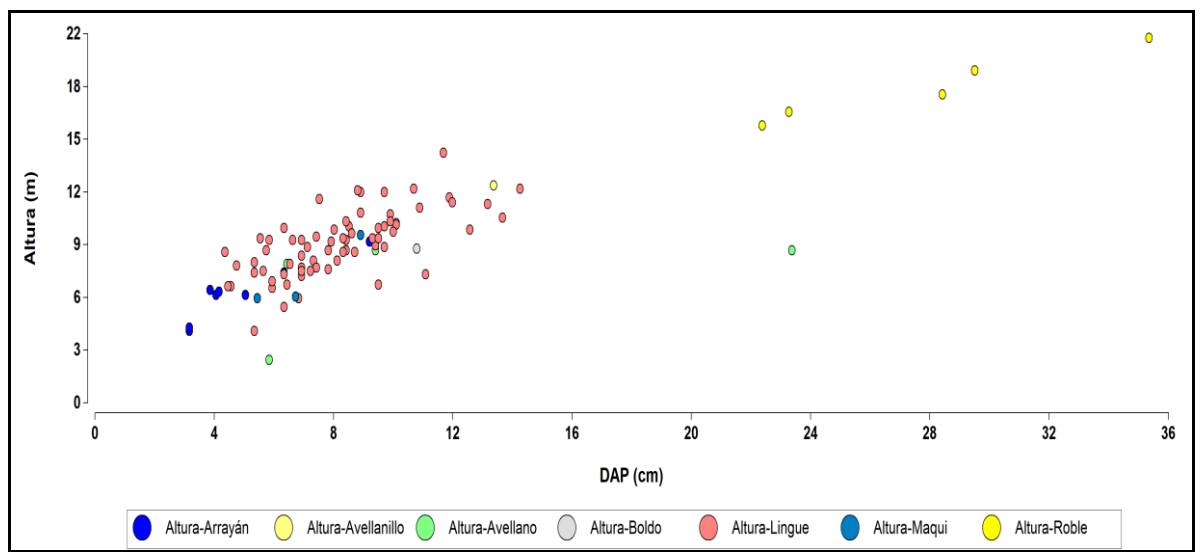

Figura $\mathrm{N}^{\circ} 3$

RELACIÓN DAP-ALTURA PARA LAS ESPECIES RODAL NACIMIENTO 1

\section{- Rodal 2. Nacimiento}

Corresponde a un rodal coetáneo, producto de una explotación y posterior incendio que sufrió el sector. Sus valores medios de DAP, altura y densidad para la especie lingue corresponden a 9,2 cm, 10,5 m y 555 árb/ha, respectivamente.

Una vista de esta unidad se presenta en la Figura $N^{\circ} 4$; la relación altura-diámetro en la Figura $N^{\circ} 5$; y la distribución espacial de los árboles de lingue en el perfil horizontal en la Figura $N^{\circ}$ 6. 


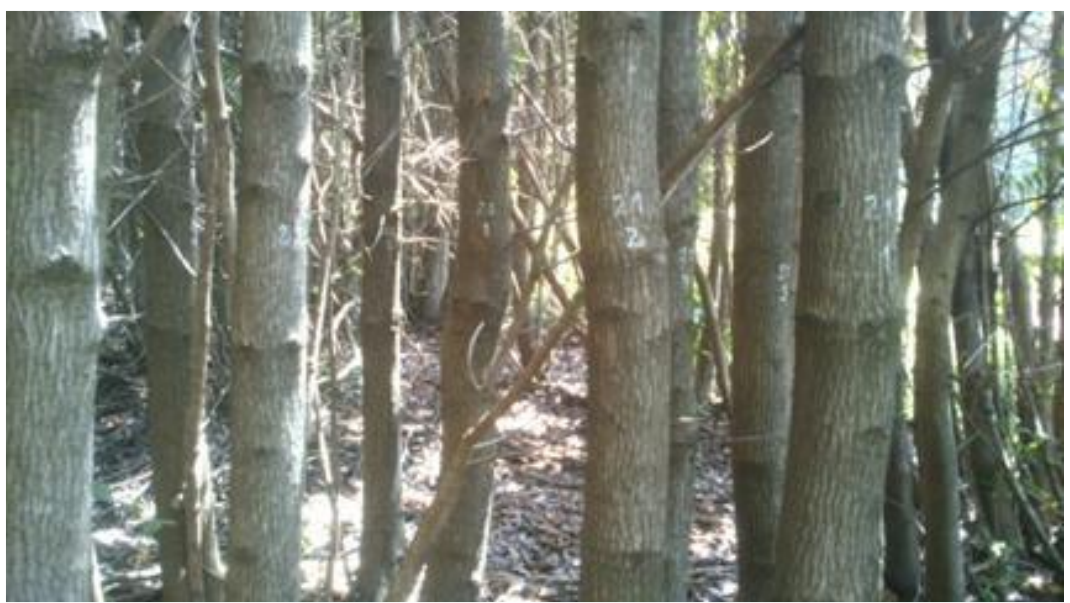

Figura $\mathrm{N}^{\circ} 4$

VISTA PARCIAL DEL RODAL DE LINGUE NACIMIENTO 2

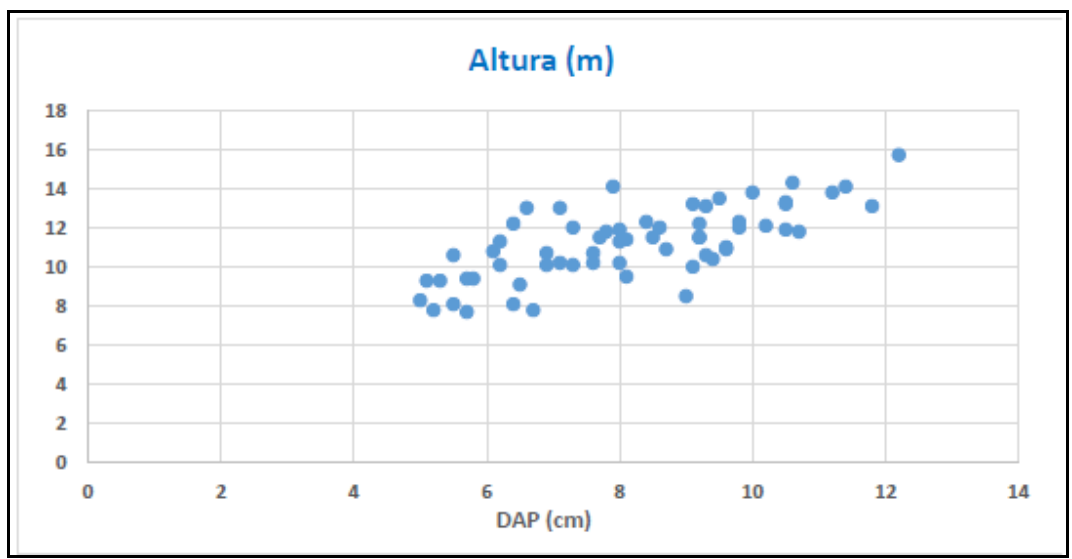

Figura $\mathbf{N}^{\circ} 5$

RELACIÓN DAP-ALTURA DE ÁRBOLES DE LINGUE MUESTREADOS EN RODAL NACIMIENTO 2 


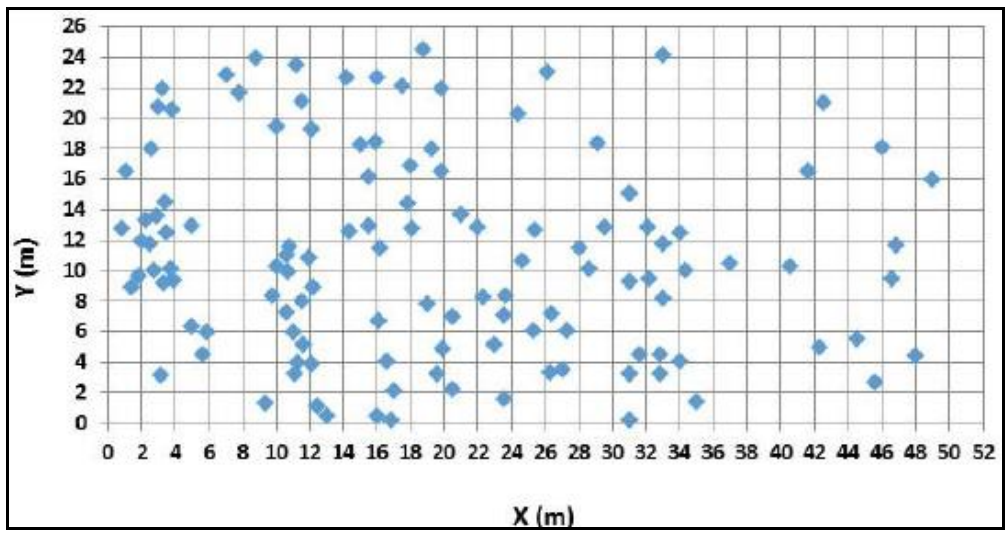

Figura $\mathrm{N}^{\circ} 6$

DISTRIBUCIÓN ESPACIAL DE ÁRBOLES DE LINGUE EN RODAL NACIMIENTO 2

\section{Rodal 3. Coihueco}

Este rodal se ubica en el predio La Esperanza, en la localidad del mismo nombre en la comuna de Coihueco, provincia del Ñuble, región del Bio Bio. Corresponde a un rodal de RobleRaulí-Avellano-Lingue, perteneciente al tipo forestal Roble-Raulí-Coigüe, sub tipo Bosques Degradados que no presenta intervenciones en al menos los últimos 30 años.

El rodal corresponde a la regeneración posterior a una fuerte intervención ocurrida durante la década de los ochenta, donde la mayoría de los arboles presentan una distribución de tipo agrupada, sin embargo, se observan también árboles establecidos por semilla o al azar. Esto estaría indicando que la mayoría de las especies existentes en el rodal presentan un modo de regeneración por claros y en ocasiones continuo si las condiciones de micro sitio les son favorables. Al respecto, se debe hacer la distinción con las especies de género Nothofagus, las cuales no presentan un modo de regeneración continua, sino que por el contrario presentan un modo de regeneración por claros y catastrófico.

Esta alta capacidad regenerativa ha permitido que las especies presenten un gran potencial y una gran plasticidad, siendo capaces de establecerse con vigorosidad, ya sea bajo sombra o a plena luz del sol. Estos dos aspectos demuestran el rango de tolerancia a la sombra que posee lingue, que constituye una importante habilidad competitiva.

La estructura diamétrica del rodal presenta una curva de tipo $\mathrm{J}$ inversa (Figura $\mathrm{N}^{\circ} 7$ ), evidenciando el reclutamiento de nuevos árboles, principalmente lingues y avellanos, que son las especies predominante en el rodal (Figura $\mathrm{N}^{\circ} 8$ ).

Existe una clara dominancia de avellano y lingue, tanto en número de árboles por hectárea como en área basal, lo que indica que las especies de mayor tolerancia a la sombra, en ausencia de perturbaciones, tenderán a dominar el rodal y convivir con intolerantes emergentes como roble o raulí en el dosel superior. Estos últimos podrán regenerar por claros producidos por la caída de árboles senescentes de mayor tamaño, produciéndose una dinámica de claros en la que podrán regenerar de manera esporádica o intermitente las especies más intolerantes. Resulta evidente que el rodal fue fuertemente intervenido, extrayéndosele la mayoría de los robles y raulíes de mejor calidad, adquiriendo una curva aplanada y de gran amplitud diamétrica, alcanzando algunos individuos la clase $90 \mathrm{~cm}$. 


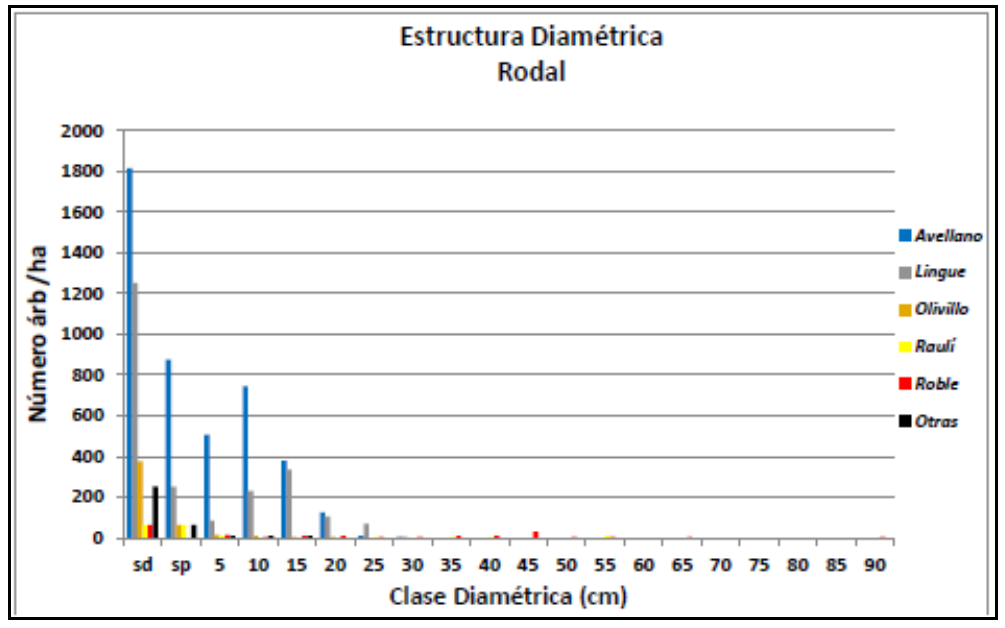

Figura $\mathrm{N}^{\circ} 7$

ESTRUCTURA DIAMÉTRICA DE LAS ESPECIES QUE COMPONEN EL RODAL DE COIHUECO
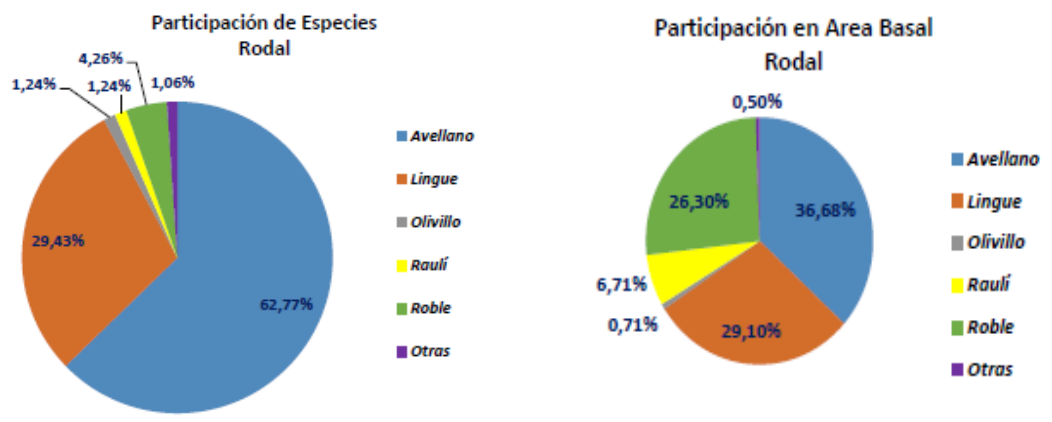

Figura $\mathbf{N}^{\circ} 8$

PARTICIPACIÓN DE ESPECIES EN LA COMPOSICIÓN DEL RODAL COIHUECO PORCENTAJE DE INDIVIDUOS (IZQ.) Y PROPORCIÓN DEL AREA BASAL (DER.)

La distribución de los individuos en el plano horizontal es del tipo agrupada, esto debido a la alta capacidad de retoñación de las especies que componen el rodal. En general, los lingues presentan este tipo de agrupación (varios retoños por tocón), sin embargo, también se encuentran individuos creciendo aislados.

La distribución vertical se caracteriza por la presencia de más de dos estratos, lo que está directamente influenciado por el sitio, por la etapa sucesional en que se encuentre el rodal y por las especies que lo constituyen.

Se observa un estrato dominante y un codominante formado principalmente por roble y raulí, sobre un estrato de árboles intermedios constituido principalmente por lingue y avellano y en 
menor proporción olivillo, laurel y avellanillo. Más abajo se pueden encontrar indistintamente los estratos de suprimidos (Figura $\mathrm{N}^{\circ}$ 9).

Resalta el volumen de madera de lingue, especie que posee posibilidades ciertas de proporcionar un valor extra al bosque, sin embargo, se debe mejorar su calidad y forma para lograr un volumen de mejor valor comercial.

Por su parte, el avellano si bien está presente en forma mayoritaria, es una especie comparativamente menos atractiva que el lingue desde el punto de vista maderero.

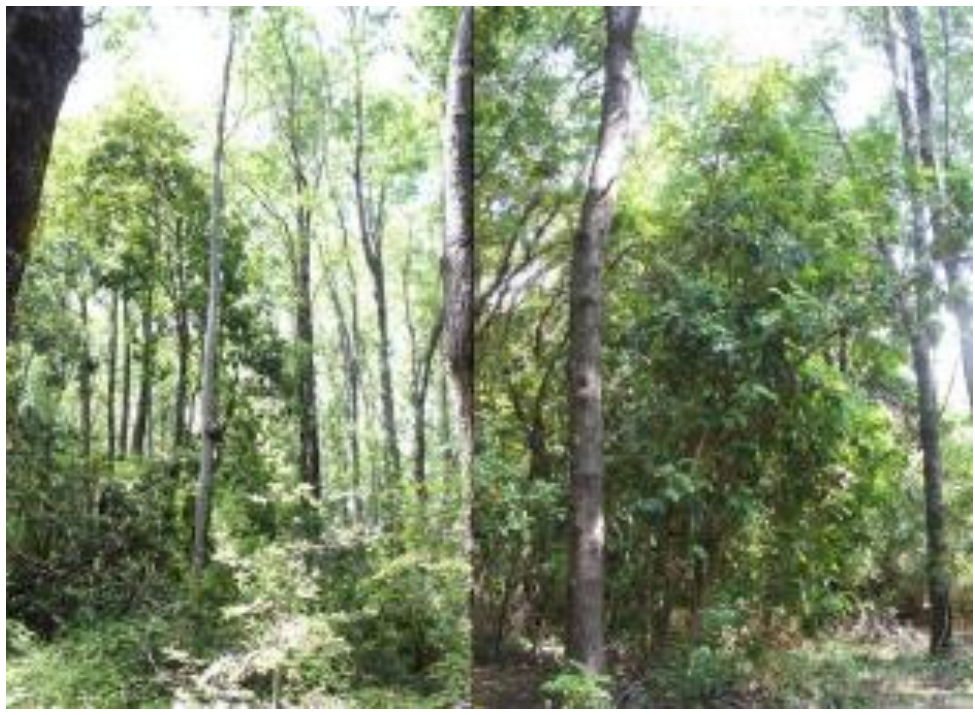

Figura $\mathbf{N}^{\circ} 9$

DISTRIBUCIÓN VERTICAL DE COIHUECO

Lingue está presente en una fase sucesional de reacción de bosques que han sido alterados por la acción antrópica, tal cual se habría señalado también en el rodal de Nacimiento. La distribución horizontal y vertical de este rodal, sugiere efectuar raleos en etapas tempranas para reducir la competencia, mejorar los incrementos en diámetro y altura, así como la calidad del bosque remanente. Respecto a los volúmenes de madera, se deben buscar alternativas para aumentar el stock futuro y la calidad de la misma mediante silvicultura intensiva.

\section{Evaluación de Ensayo de Plantación de Lingue}

El ensayo evaluado corresponde a una unidad establecida en 2014 en el sector del Museo Mapuche ubicado en la ciudad de Cañete, con el objetivo de probar el efecto de distintos tamaños de contenedor de plantas, espaciamientos y la utilización de protección individual sobre el crecimiento y supervivencia de las plantas (Pinilla y Luengo, 2016).

El diseño del ensayo consideró tres factores: (i) plantas con o sin protección con malla raschel; (ii) el uso de dos tipos de contenedores (130 y $260 \mathrm{cc}$ ); y (iii) dos espaciamientos de 
plantación (1x1m y 2x1m). Cada una de las ocho combinaciones (tratamiento) se repitió en tres parcelas de 49 plantas (Cuadro $\mathrm{N}^{\circ} 3$; Figura $\mathrm{N}^{\circ} 10$ ).

La medición evaluada corresponde a la efectuada en 2019, a la edad de cinco años.

Cuadro $N^{\circ} 3$

VARIABLES PROBADAS EN ENSAYO DE PLANTACIÓN DE LINGUE DE CAÑETE

\begin{tabular}{|c|c|c|c|c|}
\hline \multicolumn{3}{|c|}{ Factor } & \multirow{2}{*}{ Plantas } & $\begin{array}{c}\text { Superficie } \\
\text { Parcela } \\
\left(\mathrm{m}^{2}\right)\end{array}$ \\
\hline $\begin{array}{c}\text { Contenedor } \\
\left(\mathrm{cm}^{3}\right)\end{array}$ & $\begin{array}{c}\text { Espaciamiento } \\
(\mathrm{m})\end{array}$ & Protección & $\left(\mathrm{N}^{\circ}\right)$ & \begin{tabular}{c} 
\\
\hline
\end{tabular}
\end{tabular}

\begin{tabular}{|l|c|c|c|c|}
\hline 130 & $1 \times 1$ & Con & 49 & 98 \\
\hline 130 & $1 \times 1$ & Sin & 49 & 49 \\
\hline 130 & $2 \times 1$ & Con & 49 & 98 \\
\hline 130 & $2 \times 1$ & Sin & 49 & 49 \\
\hline 260 & $1 \times 1$ & Con & 49 & 98 \\
\hline 260 & $1 \times 1$ & Sin & 49 & 49 \\
\hline 260 & $2 \times 1$ & Con & 49 & 98 \\
\hline 260 & $2 \times 1$ & Sin & 49 & 49 \\
\hline
\end{tabular}

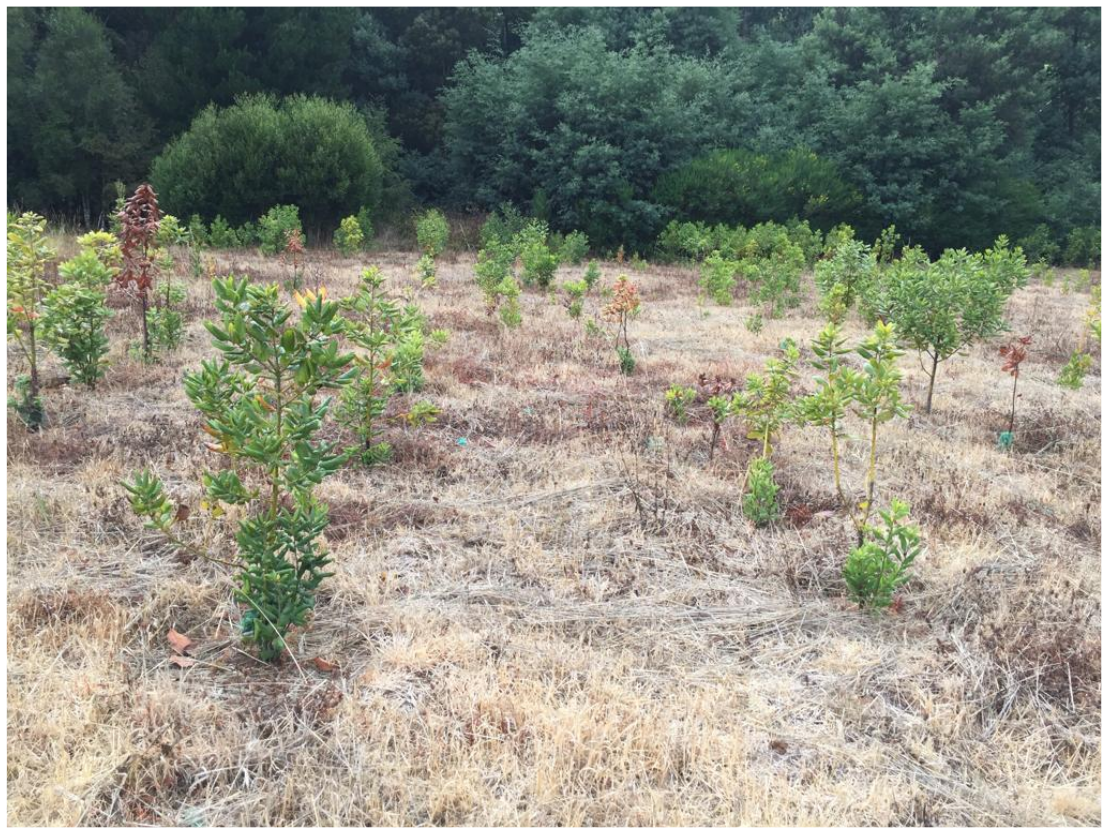

Figura $\mathbf{N}^{\circ} 10$

VISTA GENERAL DEL ENSAYO DE PLANTACIÓN DE LINGUE DE CAÑETE 
Respecto a las variables de crecimiento, el diámetro de cuello (DAC) presentan un rango entre 7 y $19 \mathrm{~mm}$, en tanto la altura fluctúa entre 72 y $118 \mathrm{~cm}$. El mejor desempeño en ambas variables se obtuvo usando contenedores de $130 \mathrm{~cm}^{3}$, protector de malla y plantando a $1 \times 1 \mathrm{~m}$ (Cuadro $\mathrm{N}^{\circ} 4$ ). En términos de supervivencia, los mayores valores se presentan en los tratamientos que utilizan contenedor de $130 \mathrm{cc}$ con protección inicial y espaciamiento de $2 \times 1 \mathrm{~m}$, todos los cuales superan el $55 \%$ (Cuadro $\mathrm{N}^{\circ} 5$ ).

\section{Cuadro $\mathrm{N}^{\circ} 4$}

ALTURA TOTAL Y DIAMETRO DE CUELLO DEL ENSAYO DE PLANTACIÓN DE LINGUE DE CAÑETE A LOS CINCO AÑOS DE EDAD

\begin{tabular}{|c|c|c|c|c|c|c|c|c|c|c|c|}
\hline \multirow{3}{*}{$\begin{array}{c}\text { Contenedor } \\
\qquad\left(\mathrm{cm}^{3}\right)\end{array}$} & \multirow{3}{*}{$\begin{array}{c}\text { Espaciamiento } \\
\text { (m) }\end{array}$} & \multirow{3}{*}{ Protección } & \multirow{3}{*}{$\mathbf{n}$} & \multicolumn{4}{|c|}{ DAC } & \multicolumn{4}{|c|}{ Altura } \\
\hline & & & & Medio & DS & Mín & Máx & Medio & DS & Mín & Máx \\
\hline & & & & \multicolumn{4}{|c|}{$(\mathrm{mm})$} & \multicolumn{4}{|c|}{$(\mathrm{m})$} \\
\hline 130 & $1 \times 1$ & Con & 43 & 18,81 & 6,97 & 3 & 32 & 118,30 & 45,37 & 20 & 173 \\
\hline 130 & $1 \times 1$ & $\operatorname{Sin}$ & 33 & 18,33 & 8,10 & 4 & 38 & 84,85 & 39,82 & 29 & 174 \\
\hline 130 & $2 \times 1$ & Con & 55 & 17,60 & 9,86 & 4 & 41 & 107,11 & 56,20 & 16 & 212 \\
\hline 130 & $2 \times 1$ & Sin & 45 & 17,71 & 7,45 & 4 & 41 & 85,20 & 36,69 & 21 & 155 \\
\hline 260 & $1 \times 1$ & Con & 13 & 11,85 & 5,57 & 4 & 21 & 73,46 & 40,90 & 21 & 140 \\
\hline 260 & $1 \times 1$ & Sin & 3 & 13,83 & 4,01 & 10 & 18 & 75,00 & 52,68 & 25 & 130 \\
\hline 260 & $2 \times 1$ & Con & 6 & 12,17 & 8,50 & 2 & 26 & 84,83 & 58,01 & 10 & 158 \\
\hline 260 & $2 \times 1$ & Sin & 1 & 7,00 & 0,00 & 7 & 7 & 72,00 & 0,00 & 72 & 72 \\
\hline
\end{tabular}

Cuadro $\mathrm{N}^{\circ} 5$

SUPERVIVENCIA DEL ENSAYO DE PLANTACIÓN DE LINGUE DE CAÑETE A LOS CINCO AÑOS DE EDAD

\begin{tabular}{|c|c|c|c|}
\hline $\begin{array}{c}\text { Contenedor } \\
\left(\mathbf{c m}^{\mathbf{3}}\right)\end{array}$ & $\begin{array}{c}\text { Espaciamiento } \\
\mathbf{( \mathbf { m } )}\end{array}$ & Protección & $\begin{array}{c}\text { Supervivencia } \\
(\%)\end{array}$ \\
\hline 130 & $1 \times 1$ & Con & 57,3 \\
\hline 130 & $1 \times 1$ & Sin & 44,0 \\
\hline 130 & $2 \times 1$ & Con & 73,3 \\
\hline 130 & $2 \times 1$ & Sin & 60,0 \\
\hline 260 & $1 \times 1$ & Con & 17,3 \\
\hline 260 & $1 \times 1$ & Sin & 4,0 \\
\hline 260 & $2 \times 1$ & Con & 8,0 \\
\hline 260 & $2 \times 1$ & Sin & 1,3 \\
\hline
\end{tabular}

El efecto del tipo de contenedor, espaciamiento y uso de malla protectora sobre la altura y diámetro de cuello de las plantas se resume en los Cuadros $\mathrm{N}^{\circ} 6, \mathrm{~N}^{\circ} 7$ y $\mathrm{N}^{\circ} 8$. En ellos se observa que, tanto en el DAC como en las alturas, existen diferencias significativas, siendo el contenedor de $130 \mathrm{~cm}^{3}$ el que presenta los mayores valores (Cuadro $\mathrm{N}^{\circ} 6$ ). El espaciamiento no evidencia efecto sobre el crecimiento, al menos a la edad de evaluación (Cuadro $\mathrm{N}^{\circ} 7$ ). En tanto el uso de malla protectora solo muestra efectos significativos para la variable altura, que paradójicamente resulta menor al usar malla, mientras que no exhibe efecto sobre el diámetro (Cuadro $\mathrm{N}^{\circ} 8$ ). 
Cuadro $\mathrm{N}^{\circ} 6$

RESULTADOS SEGÚN TIPO DE CONTENEDOR

\begin{tabular}{|c|c|}
\hline $\begin{array}{c}\text { Contenedor } \\
\left(\mathbf{c m}^{\mathbf{3}}\right)\end{array}$ & Medias \\
\hline & $\begin{array}{c}\text { DAC } \\
\text { (mm) }\end{array}$ \\
\hline \multirow{2}{*}{260} & $11,98 \mathrm{~A}$ \\
130 & $18,06 \mathrm{~B}$ \\
\hline & Altura \\
& (cm) \\
\hline \multirow{2}{*}{260} & $76,57 \mathrm{~A}$ \\
130 & $100,07 \mathrm{~B}$ \\
\cline { 2 - 2 }
\end{tabular}

Cuadro $\mathrm{N}^{\circ} 7$

RESULTADOS SEGÚN ESPACIAMIENTO INICIAL

\begin{tabular}{|c|c|}
\hline $\begin{array}{c}\text { Espaciamiento } \\
(\mathrm{m})\end{array}$ & Medias \\
\hline
\end{tabular}

\begin{tabular}{|c|c|}
\hline & $\begin{array}{c}\text { DAC } \\
(\mathbf{m m})\end{array}$ \\
\hline \multirow{2}{*}{$2 \times 1$} & $17,24 \mathrm{~A}$ \\
$1 \times 1$ & $17,49 \mathrm{~A}$ \\
\hline
\end{tabular}

\begin{tabular}{|c|c|}
\hline \multirow{2}{*}{$2 \times 1$} & $\begin{array}{c}\text { Altura } \\
\text { (cm) }\end{array}$ \\
\cline { 2 - 2 } $1 \times 1$ & $96,32 \mathrm{~A}$ \\
$98,55 \mathrm{~A}$ \\
\hline
\end{tabular}

Cuadro $\mathrm{N}^{\circ} 8$

RESULTADOS SEGÚN PROTECCIÓN INICIAL

\begin{tabular}{|c|c|}
\hline Protección & Medias \\
\hline \multirow{4}{*}{ Con } & $\begin{array}{c}\text { DAC } \\
\text { (mm) }\end{array}$ \\
\cline { 2 - 2 } Sin & $17,33 \mathrm{~A}$ \\
\hline \multicolumn{2}{|c|}{$17,69 \mathrm{~A}$} \\
\hline \multirow{3}{*}{ Con } & Altura \\
Sin & (cm) \\
\cline { 2 - 2 } & $84,52 \mathrm{~A}$ \\
\end{tabular}

Considerando todas las combinaciones de tratamientos en forma independiente, se observa que. tanto para altura como para diámetro. se configuran dos grupos diferentes (Cuadro $\mathrm{N}^{\circ}$ $9)$. 
En general se confirma que los tratamientos que utilizaron contenedores de $130 \mathrm{~cm}^{3}$ presentaron los mejores resultados. En cuanto al espaciamiento inicial o la utilización de una malla de protección inicial no se generaron diferencias significativas.

Por el contrario, en el DAC los menores resultados se presentaron con los contendores de $260 \mathrm{~cm}^{3}$, siendo estadísticamente diferentes de los resultados obtenidos con el contenedor de $130 \mathrm{~cm}^{3}$ en la evaluación del año 2019. Respecto de la altura de las plantas, se generan también dos grupos de resultados estadísticamente diferentes, donde nuevamente los tratamientos que utilizaron contenedores de $130 \mathrm{~cm}^{3}$ presentaron los mejores resultados. El espaciamiento inicial no generó diferencias significativas, lo que sí aconteció con la utilización de malla inicial como protección de las plantas. En la altura, los menores resultados se presentaron con los contendores de $260 \mathrm{~cm}^{3}$ en la evaluación del año 2019.

\section{Cuadro $N^{\circ} 9$}

DIÁMETROS Y ALTURAS SEGÚN TRATAMIENTO INDIVIDUAL

\begin{tabular}{|c|c|c|c|c|}
\hline $\begin{array}{c}\text { Contenedor } \\
\left(\mathrm{cm}^{3}\right)\end{array}$ & $\begin{array}{c}\text { Espaciamiento } \\
(\mathrm{m})\end{array}$ & Protección & $\begin{array}{c}\text { DAC } \\
(\mathrm{mm})\end{array}$ & $\begin{array}{c}\text { Altura } \\
(\mathrm{cm})\end{array}$ \\
\hline
\end{tabular}

\begin{tabular}{|c|c|c|c|c|c|c|}
\hline 130 & $1 \times 1$ & Con & 18,8 & $\mathrm{~B}$ & 118,3 & $\mathrm{~B}$ \\
\hline 130 & $1 \times 1$ & Sin & 18,3 & $\mathrm{~B}$ & 84,9 & $\mathrm{AB}$ \\
\hline 130 & $2 \times 1$ & Con & 17,6 & $\mathrm{~B}$ & 107,1 & $\mathrm{~B}$ \\
\hline 130 & $2 \times 1$ & Sin & 17,7 & $\mathrm{~B}$ & 85,2 & $\mathrm{AB}$ \\
\hline 260 & $1 \times 1$ & Con & 11,9 & $\mathrm{AB}$ & 73,5 & $\mathrm{~A}$ \\
\hline 260 & $1 \times 1$ & Sin & 13,8 & $\mathrm{AB}$ & 75,0 & $\mathrm{~A}$ \\
\hline 260 & $2 \times 1$ & Con & 12,2 & $\mathrm{AB}$ & 84,8 & $\mathrm{AB}$ \\
\hline 260 & $2 \times 1$ & Sin & 7,0 & $\mathrm{~A}$ & 72,0 & $\mathrm{~A}$ \\
\hline
\end{tabular}

\section{CONCLUSIONES}

Respecto de los rodales naturales de lingue, los resultados obtenidos señalan que es necesario desarrollar investigaciones sobre el manejo de estos en diferentes condiciones de sitio en términos de raleos en renovales y en casos necesarios plantaciones de enriquecimiento en los tipos forestales en los que participa la especie, técnicas que pueden ofrecer en el mediano plazo bosques comerciales nuevamente.

En cuanto a las plantaciones de lingue se puede concluir que:

Existen diferencias de crecimiento y supervivencia entre los tratamientos utilizados, con un claro efecto positivo del tipo de contenedor, el cual genera un efecto en el DAC, la altura y la supervivencia de las plantas.

El mayor efecto en la supervivencia se obtuvo con contenedor de $130 \mathrm{~cm}^{3}$, un espaciamiento inicial de 2X1m y con protección inicial (malla), las que alcanzaron un promedio de $73 \%$, en comparación a la supervivencia menor al $10 \%$ que se observó en los tratamientos que utilizaron un contenedor de $260 \mathrm{~cm}^{3}$ y un espaciamiento de $2 \times 1 \mathrm{~m}$.

Los mayores valores en DAC y altura se obtuvieron en la combinación de un contenedor de $130 \mathrm{cc}$, un espaciamiento de 1x1m y con una protección individual (malla).

Es necesario seguir mantener el ensayo y continuar sus evaluaciones para determinar 
variaciones en el crecimiento de las plantas y la permanencia en el tiempo de los efectos de los distintos factores utilizados.

La mantención del ensayo debe considerar la labor de podas de formación, estimulación de dominancias apical y limpieza.

\section{REFERENCIAS}

Pinilla, J. C. y Navarrete, T., 2013. Reporte de prácticas silvícolas en bosques naturales de la zona centro sur, La situación de Lingue y Salix chileno en la región del Bio Bio para su uso potencial en bosques plantados. Proyecto 3: Investigación forestal manejo y recuperación de bosque nativo. INFOR - MINAGRI 2013. 94p.

Pinilla S., J.C.; Luengo V., K. y Navarrete T., M., 2015. Lingue (Persea lingue (R. et Pav.) Nees ex Kopp) en la región del Bio Bio. Revista Ciencia e Investigación Forestal, CIFOR, v.21(2), Pág.:71-96

Pinilla, J.C. y Luengo, K., 2016. Antecedentes generales para el establecimiento de Lingue. Ficha divulgativa INFOR. 